\title{
FACTORES DE LA CALIDAD DE CARTERA DE CRÉDITOS EN LA RENTABILIDAD DE UNA CAJA MUNICIPAL DE AHORRO Y CRÉDITO
}

\section{FACTORS OF LOAN PORTFOLIO QUALITY ON THE PROFITABILITY OF A MUNICIPAL SAVINGS AND LOAN INSTITUTION.}

Sandra Cristina Cueto Sanchez ${ }^{1}$

https://orcid.org/0000-0003-3578-2260 scueto0809@gmail.comi

Ruben Dario Ticlavilca Forlong ${ }^{2}$

https://orcid.org/0000-0002-4767-6046

rubticlavilcaf@virtual.upt.pe

Aceptado:01/12/2020 Publicado online:30/09/2021

\begin{abstract}
RESUMEN
El objetivo de esta investigación fue establecer la influencia de los factores relacionados a la calidad de cartera de créditos en la rentabilidad de la Caja Municipal de Ahorro y Crédito De Tacna S.A. El tipo de investigación fue básico; el diseño fue no experimental, longitudinal; el nivel fue causal-explicativo; la mues tra estuvo compuesta por los documentos históricos de la Caja Municipal de Ahorro y Crédito De Tacna S.A. emitidos por la SBS, del periodo 2017 - 2018, que contuvieron información acerca de las variables internas de la entidad, además de los documentos históricos emitidos INEI, acerca de las variables macroeconómicas; para la evaluación de las variables se halla ron los esta dís ti cos descriptivos e inferenciales. Entre los resultados más resal tantes se obtuvo que la rentabilidad inició muy cerca de $0 \%$ al iniciar el periodo 2017, elevándose hasta diciembre del periodo 2017 y cayendo estrepitosamente; dicha tendencia fue similar al iniciary finalizar el periodo 2018. No se pudo realizar una regresión lineal, pero queda la posibilidad de aplicar una regresión multivariada que permita agrupar las variables dentro de un modelo. Si se desarrolla un model o quitando algunas variables, el aporte del modelo no sería sustancial. Sin embargo, es posible afirmar que existe una alta influencia de las Colocaciones por tipo de crédito en la Rentabilidad dela Caj a Municipal de Ahorro y Crédito De Tacna S.A. con un coeficiente de determi nación $\left(r^{2}\right)$ promedio de 0,6133.
\end{abstract}

Palabras clave: rentabilidad, morosidad, colocaciones, créditos, calidad de cartera

\footnotetext{
1 Universidad Privada de Tacna.

2 Universidad Privada de Tacna, Facultad de Ciencias empresariales.
} 


\begin{abstract}
The quality of the Ioan portfolio in the profitability of the Caja Municipal de Ahorro y Crédito De Tacna S.A. The type of investigation was basic; the design was non-experimental, longitudinal; the level was causal - explanatory; The sample consisted of the historical documents of the Caja Municipal de Ahorro y Crédito De Tacna S.A. issued by the SBS, for the period 2017 - 2018, which contained information about the entity's internal variables, in addition to the historical documents issued INEI, about macroeconomic variables; Descriptive and inferential statistics were found for the evaluation of the variables. Among the most outstanding results, it was obtained that profitability started very close to $0 \%$ at the beginning of the 2017 period, rising until December of the 2017 period and falling sharply; This trend was similar at the beginning and end of the 2018 period. A linear regression could not be performed, but there is still the possibility of applying a multivariate regression that allows the variables to be grouped within a model. If a model is developed by removing some variables, the contribution of the model would not be substantial. However, it is possible to affirm that there is a high influence of Placements by type of credit on the Profitability of the Caja Municipal de Ahorro y Crédito De Tacna S.A. with an average coefficient of determination ( $r 2)$ of 0.6133 .
\end{abstract}

Keywords: profitability, delinquency, loans, loans, portfolio quality.

\title{
INTRODUCCIÓN
}

La presente investigación se realiza porque se desea brindar un aporte a la Caja Municipal de Ahorro y Crédito De Tacna S.A.; la cual es una entidad representativa de la Región de Tacna y del Sur del Perú, centrando la investigación en la Rentabilidad de la misma. Dicha rentabilidad es influenciada por diversas variables; que la entidad maneja dentro de sugestión y otras que no maneja, como es el caso de las variables macroeconómicas del Perú. Este tema, despierta el interés del personal que dirige y labora dentro de esta entidad, de las autoridades que tienen cierta injerencia, de los ahorristas y prestatarios que interactúan con la entidad desde su creación. Merchán (2016) dio a conocer que los prestatarios que residen más lejos de los centros urbanos, muestran que las probabilidades de incumplimiento se reducen. Así mismo, las mujeres con ingreso bajo y con un nivel educativo con el grado de bachiller cuentan con una probabilidad menor de incumplimiento. Por otra parte, se halló que un número mayor de instituciones financieras en una zona, incrementa la probabilidad de incumplimiento. Giraldo (2010) resalta que la cartera vencida es sensible a los choques de la cartera total, de forma directa y positiva para el sistema financiero agregado y algunas de las entidades analizadas; Lozano (2007) hace hincapié en la relación directa entre la eficiencia del personal y la cartera vencida. Es preciso indicar que las entidades financieras con más de 10000 clientes, no son sensibles a la influencia de la eficiencia del personal. Además, se dio a conocer la importancia de la uniformidad en los procesos de dación de créditos. Acosta \& Sánchez (2016) mostró que la calidad de cartera es fundamental para el análisis de la gestión financiera, pues repres enta la mayor fuente de riesgo para las entidades financieras; entre los factores determinantes resalta la cartera de riesgo, gestión de créditos, índices de morosidad, gastos en provisiones. Entre las alternativas para controlar la mora, se encuentran los convenios con terceros con la finalidad de congelar las deudas. Para Gomez (2011) los principales factores que generan morosidad son la mala evaluación económica y financiera, el 
uso inadecuado de los créditos por parte de los clientes, falta de seguimiento del crédito. Supo (2015) dio a conocer los factores que afectaron la calidad de la cartera: número de entidades financieras con las que el cliente mantiene obligaciones financieras, ratio de endeudamiento, plazo otorgado al crédito, estado civil del prestatario, tiempo de constitución del negocio, destino del crédito. Por otro lado, el incumplimiento del plan de negocios eleva la probabilidad de morosidad en un 9,2\%; el nivel de endeudamiento afecta en un 7,3\% la calidad de cartera, un mayor tiempo de constitución del negocio disminuye en un 0,1\% la morosidad. Calloapaza (2017) resalta que la evolución de la morosidad de la CMAC De Tacna S.A. correspondientes al periodo 2010 - 2014, ha sido desfavorable; pues se demostró un crecimiento notorio equivalente al $255 \%$. Martínez (2006) identificó las causas del incremento del Riesgo Crediticio en CMAC Tacna; por ejemplo, las colocaciones al sector agrícola, que mantiene índices elevados de morosidad afectando directamente la rentabilidad y utilidades de la organización. Otra de las causas es el sobreendeudamiento que genera el incumplimiento en sus obligaciones financieras. Para Cornejo (2017) el crecimiento económico influye de forma inversa en las provisiones, disminuyéndolas; la volatilidad del tipo de cambio no influye en las provisiones de las Cajas Municipales de Ahorro y Crédito en el Perú, porque los préstamos en moneda extranjera son mínimos; la inflación influye de manera inversa en la rentabilidad; las provisiones afectan de manera inversa en la rentabilidad.

A nivel nacional, las CMACs juegan un papel preponderante, al abarcar una parte importante en la dación de créditos a las pequeñas y micro empresas; permitiendo que muchas de estas empresas se apalanquen financieramente y generen ingresos que aporten a la economía del país en muchos sectores. Las CMACs, también han servido como pilar de la descentralización con respecto a la capital (Lima), llevando recursos económicos a las personas emprendedoras, que requieren de los diferentes servicios bancarios; de esta manera, las localidades alejadas de la capital han podido desarrollarse. Dentro del sistema financiero peruano, existen 54 empresas de operaciones múltiples, dentro de estas, 11 empresas son CMAC; las cuales tienen activos equivalentes a S/. 27988 millones de soles, representando un $6,17 \%$ de participación del mercado financiero nacional. Es preciso resaltar que, dentro de la estructura de créditos directos, al finalizar el periodo 2018 , el $9 \%$ de créditos es destinado a la pequeña empresa y el $14 \%$ es destinado a la microempresa, siendo estos segmentos los principales para las CMAC (SBS, 2019). Por otro lado, los reportes de estabilidad financiera del periodo 2017 y 2018 señalan que, los indicadores de rentabilidad en los dos últimos periodos se han disminuido debido a una reducción de las tasas de interés activas y al aumento de las tasas de interés pasivas (BCRP, 2018).

Se incrementó la base patrimonial al capitalizar las utilidades generadas, así como la obtención de préstamos subordinados de fondos internacionales especializados. Así mismo, en el mismo periodo las colocaciones se desaceleraron, la morosidad incrementó y se realizó un mayor gasto en provisiones (BCRP, 2019). Como medida correctiva, las CMAC mejoraron su política crediticia, para contener el incremento de la morosidad y mejorar la calidad de su cartera de créditos, a la vez, han disminuido su exposición a segmentos de mercado de alto riesgo; sin embargo, aún se observa un bajo crecimiento en las colocaciones. Es imprescindible tener ciertas consideraciones al momento de hablar de la rentabilidad de las CMAC, pues no solo están sujetas al desarrollo de sus actividades financieras propias, pues existen variables exógenas que influyen en los resultados, como las macroeconómicas, por mencionar algunas de ellas: el PBI, el empleo, el tipo de cambio real, la inflación (Aparicio \& Moreno, 2011). A la vez, los bancos segmentan su mercado a la demanda de menor riesgo; 
dejando de lado las pequeñas o micro empresas con problemas de información y altos costos fijos para transacciones de montos pequeños, dicho mercado es atendido por las CMAC.

Al cierre del 2017 se maximizó la utilidad y se elevaron las expectativas para el siguiente periodo, pero las provisiones de cartera de créditos fueron consideradas como insuficientes, requiriendo un plan de fortalecimiento en el corto plazo (Equilibrium, 2018); sin embargo, al cierre del periodo 2017 cuenta con el 3,92\% de créditos directos, 4,16\% de depósitos totales, 4,22\% de patrimonio con respecto al Mercado Micro Financiero de los 8 departamentos de cobertura. En el periodo 2018, se observan mejoras en sus procesos y la productividad de sus analistas, debido a una mejora en su sistema de incentivos; sin embargo, la morosidad ha incrementado y está por encima del promedio de CMAC, lo cual está vinculado con el incremento de la cartera pesada; otro hecho importante es la recategorización de créditos normales a dudosos y simultáneamente registrados como operaciones refinanciadas en lugar de reprogramadas (Equilibrium, 2019); al cierre del periodo 2018 cuenta con el 3,90\% de créditos directos, 4,01\% de depósitos totales, 4,20\% de patrimonio. Un punto importante es que, al comparar los periodos 2017 y 2018 , el patrimonio solo creció un $9,13 \%$ debido que se limitó el otorgamiento y seguimiento de créditos en la agencia del departamento de Arequipa, con respecto al Mercado Micro Financiero de los 8 departamentos de cobertura. Las colocaciones en este periodo se redujeron en un $8,2 \%$, las colocaciones destinadas a pequeñas empresas y micro empresas se redujeron; mientras tanto, las colocaciones a créditos de consumo se incrementaron. La morosidad incrementó en la cartera atrasada $(0,31 \%)$ y la cartera problema $(1,66 \%)$; cabe resaltar que la mora del sector también incrementó, pero la mora presentada por CMAC Tacna sigue estando por encima del sector. Las provisiones totales se elevaron en un 17,83\%, equivalente a S/. 69,8 millones de soles. En conjunto, estos cambios financieros, repercuten en la rentabilidad de CMAC Tacna; el retorno sobre activos promedio se redujo de $8,16 \%$ a $7,66 \%$; el retorno sobre el patrimonio promedio también se redujo, de 1,18\% a 1,08\%.

Por la información descrita anteriormente, a través de esta investigación, se busca identificar tanto las variables macro económicas como las variables internas, relacionadas con la calidad de la cartera de crédito de la Caja Municipal de Ahorro y Crédito de Tacna S.A. durante el período 2017-2018. De esta forma, se tendría un mayor conocimiento de la situación financiera en la que se encuentra la organización; reduciendo de alguna forma los riesgos, al estar preparados ante alguna variación negativa de la tendencia de las variables internas o externas a la entidad, que afectaría la rentabilidad. Entonces, ¿En qué medida los Factores relacionados a la calidad de cartera de créditos influyen en la rentabilidad de la Caja Municipal de Ahorro y Crédito De Tacna S.A., periodo 2017 - 2018?

\section{OBJETIVO}

Establecer la influencia de los factores relacionados a la calidad de cartera de créditos en la rentabilidad de la Caja Municipal de Ahorro y Crédito De Tacna S.A., periodo 2017 - 2018.

\section{METODOLOGÍA}

El tipo de investigación de este estudio es del tipo básico no experimental de corte longitudinal esto significa que se busca la comprensión de los aspectos fundamentales de las variables observadas o de las relaciones establecidas entre ellas (RENACYT, 2005). 
La investigación se desarrolló en base a los documentos históricos de la Caja Municipal de Ahorro y Crédito De Tacna S.A. (CMAC Tacna), correspondientes al periodo 2017 - 2018. La población y muestra del presente trabajo de investigación está compuesta por el registro de los reportes desarrollados por CMAC Tacna y expuesto por el portal de la Superintendencia de Banca, Seguros y AFP. Siendo un total de 24 reportes, por cada una de las variables estudiadas. También, estuvo compuesta por los registros del INEI, que contiene un total de 24 reportes por cada una de las variables estudiadas. El procedimiento llevado a cabo es el siguiente: Recopilación de información de las fuentes secundarias, agrupación de la información en un solo formato, procesamiento de los datos, descripción estadística de las variables, correlación entre las variables y a nálisis del comportamiento de las variables. Para el desarrollo del estudio se utilizó la técnica de Revisión Documental; se escogió esta técnica, porque lo que se busca es tomar la información cuantitativa de los registros provistos por la SBS y el INEI, para analizarla a través de la estadística descriptiva e inferencial (Vara, 2010).

El instrumento seleccionado, de acuerdo a la técnica escogida, es la Guía de Revisión Documental; este instrumento sirve para ubicar de manera pertinente la información acopiada, analizando la información registrada en los documentos (Vara, 2010).

Para lograr recopilar la información se realizó una búsqueda de la información a través de los portales: Superintendencia de Banca, Seguros y AFP; Instituto Nacional de Estadística e Informática. Se descargó todos los archivos que contenían la información de cada una de las variables en estudio. Una vez tabulados los datos se procedió a pasar los datos a una matriz en Excel. Más adelante, se vació toda la data en el programa IBM SPSS, con su respectiva nomenclatura (Vara, 2010). El análisis e interpretación de los datos, fue aplicando la estadística descriptiva e inferencial. La estadística inferencial por su parte, permite realizar las evaluaciones para determinar la normalidad de las variables, establecer el estadístico adecuado y poder realizar pronósticos (Arriaza, 2006).

\section{RESULTADOS}

Figura 1

Morosidad por cada tipo de crédito y el total de créditos

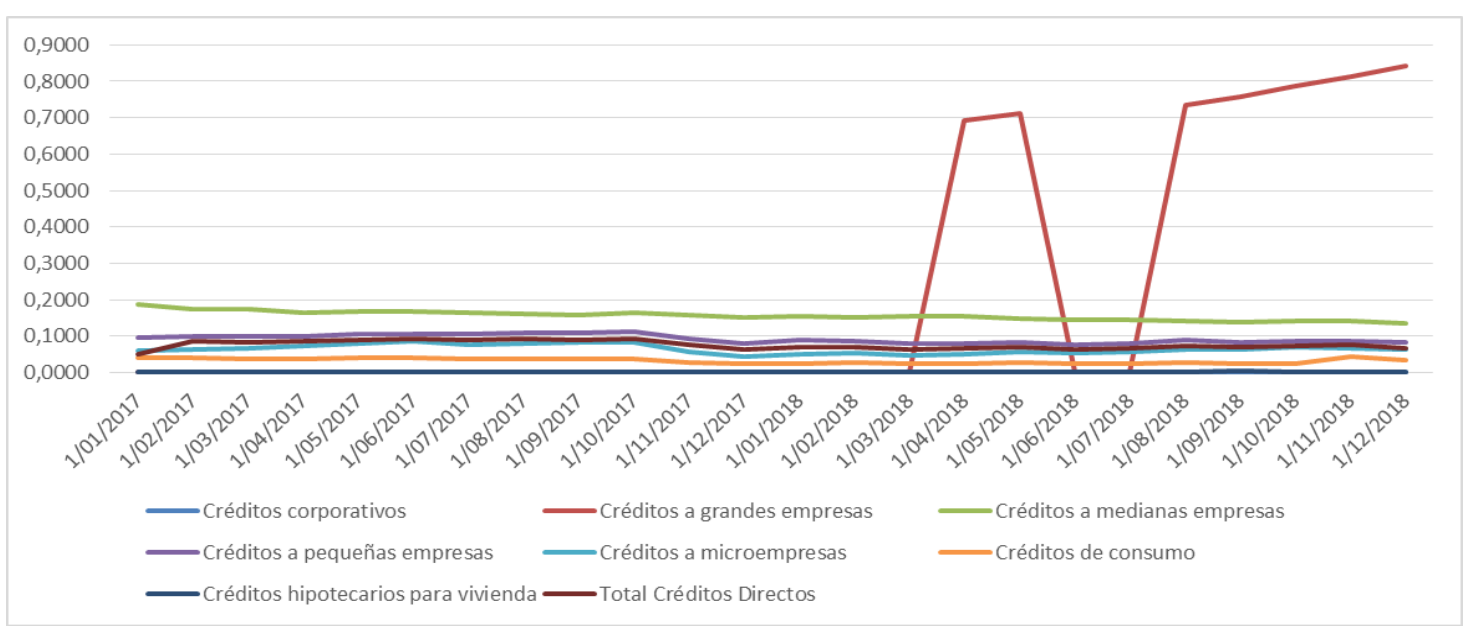




\section{Factores relacionados a la calidad de cartera de créditos: Análisis de la Morosidad Por Tipo de Crédito}

De acuerdo a la figura 1, la morosidad del crédito a medianas empresas se ha reducido paulatinamente. En el caso de la Morosidad de los Créditos a grandes empresas en marzo del 2018 se elevó y se mantuvo por dos meses, luego bajó casi completamente en junio del 2018; luego, en julio del 2018 se elevó de forma intempestiva hasta agosto del mismo periodo, para continuar con un crecimiento hasta finalizar el periodo 2018. Así mismo, la morosidad de los demás tipos de créditos tiene una leve tendencia a bajar, por debajo del $10 \%$.

\section{Análisis de las Provisiones Por Tipo De Crédito}

Los resultados de la figura 2, muestran que las Provisiones de los Créditos a grandes empresas se han reducido paulatinamente; sin embargo, a partir de junio del periodo 2018 varía la categoría de dudoso y luego lo hace la categoría de deficiente; esto como consecuencia de la clasificación de riesgo del deudor por atrasos mayores a 60 días para la Categoría Deficiente y atrasos mayores a 120 días de incumplimiento de pago para la categoría Dudoso.

Los resultados de la figura 3, muestran que la constitución de Provisiones específicas (por clasificación de CPP a Per) siempre representan el mayor gasto según la relación de a mayor clasificación - mayor provisión y, en este contexto al alcanzar la clasificación pérdida, el saldo del capital de los créditos deben estar coberturados al $100 \%$ por provisiones a menos que medien garantías reales del tipo preferente; es así que, en enero del periodo 2017 dichas provisiones ascendían a 3651 126,93 y en diciembre del periodo 2018 las provisiones en esta categoría ascendían a 5740 229,69; por ello es posible afirmar que las provisiones de los Créditos a medianas empresas en la categoría de Pérdida han incrementado y posiblemente, continúen en esa tendencia.

Los resultados de la figura 4 claramente muestran que durante el ejercicio 2017 se registró un mayor volumen de créditos con incumplimientos de pago expresados en una curva más alta en provisiones en clasificación Pérdida; es así que, en enero del 2017 dichas provisiones ascendían a 27318 982; sin embargo a inicios del cuarto trimestres del mismo periodo estas provisiones se incrementaron a 36972 144; mientras que, durante el ejercicio 2018 la curva se atenúa para continuar con una tendencia ondulatoria hasta finalizar el ejercicio. Es preciso indicar que las colocaciones de las pequeñas empresas han incrementado en dicho periodo, sin embargo, las provisiones en las otras categorías, no han incrementado en la misma proporción; por ello, se considera que se ha realizado una buena gestión por parte de la entidad.

\section{Provisiones de los Créditos a microempresas}

Los resultados de la figura 5, muestran que las Provisiones de los Créditos a microempresas en la categoría de Pérdida se han mantenido elevadas; así mismo, en enero del periodo 2017 dichas provisiones ascendían a 7558054 y en diciembre del periodo 2018 las provisiones en esta categoría ascendían a 7297 946. Sobre las variaciones de provisiones en clasificación pérdida observadas en las curvas obedecen a la ejecución de la política de castigos de créditos incobrables, procediendo a retirar el saldo de los registros contables. Es preciso indicar que las colocaciones de las microempresas se han reducido levemente en dicho periodo. Por otra parte, las provisiones en las demás categorías, no mostraron cambios; así mismo, dichas provisiones no se elevaron por encima de los 200000 soles en el tipo de Créditos a microempresas. 


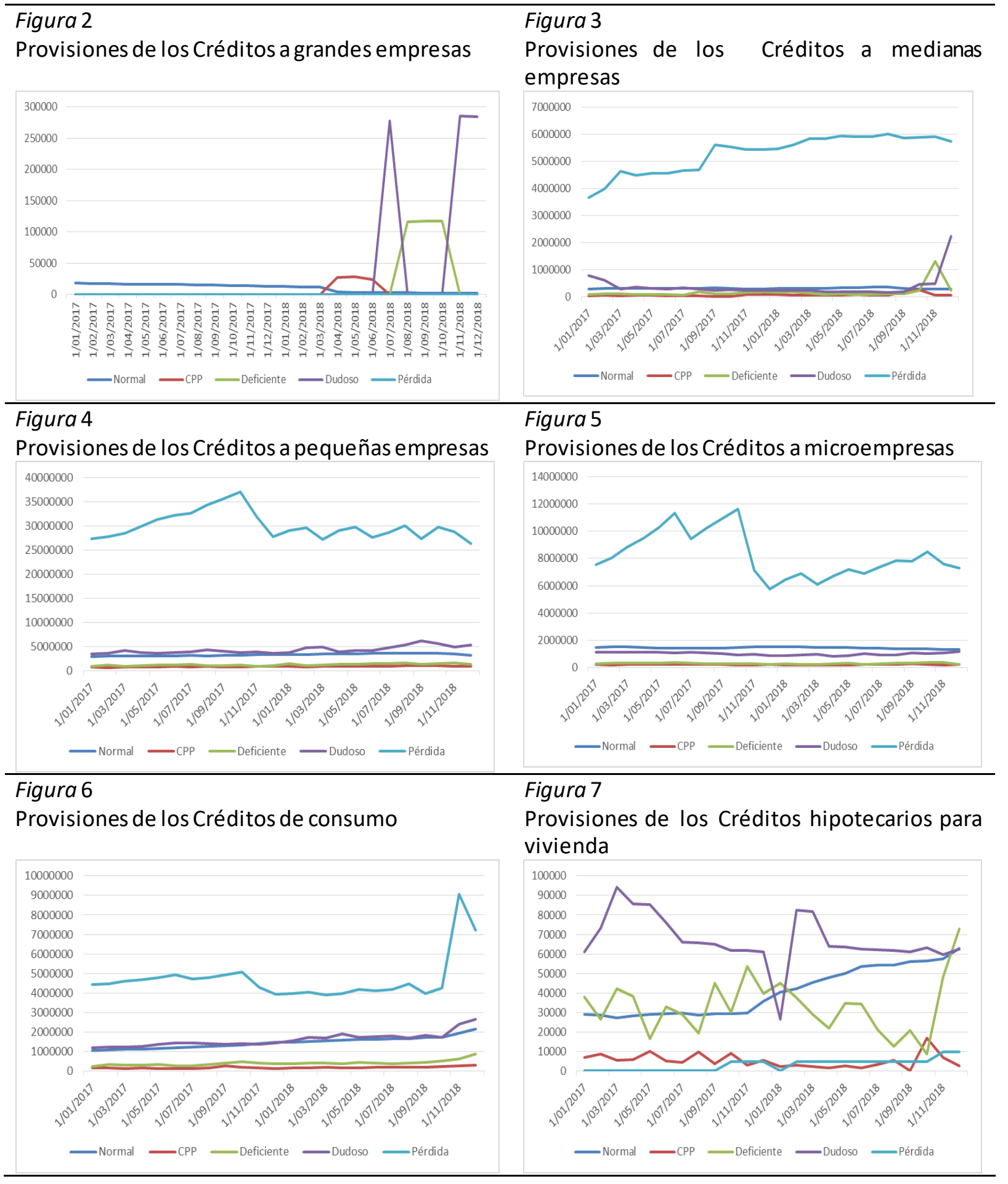

\section{Provisiones de los Créditos de consumo}

Los resultados de la figura 6, muestran que las Provisiones de los Créditos de consumo en la categoría de Pérdida se han mantenido elevada por encima de los demás créditos; así mismo, en enero del periodo 2017 dichas provisiones ascendían a 4424318,52 y en noviembre del 2018 las provisiones en esta categoría ascendían a 9077 345,28 mostrando una variación 
considerable; sin embargo en diciembre del periodo 2018 las provisiones en esta categoría ascendían a 7203 312,92 mostrando una gran elevación en estos dos periodos.

Las provisiones en la categoría de normal y dudoso, muestran una tendencia creciente leve, pues en enero del periodo 2017 dichas provisiones apenas superaban el millón de soles y en diciembre del periodo 2018 las provisiones en esta categoría superaban los dos millones de soles. Por otra parte, las provisiones en las demás categorías, mostraron cambios leves; así mismo, dichas provisiones continuaron por debajo del millón de soles.

\section{Provisiones de los Créditos hipotecarios para vivienda}

Los resultados de la figura 7, muestran que las Provisiones de los Créditos hipotecarios para vivienda; dentro de la categoría Dudoso han mostrado diversas variaciones y a pesar de no mostrar una tendencia, se puede observar que al iniciarel periodo 2017 y al finali zar el periodo 2018 el monto fue de 60000 aproximadamente. Dicha categoría se ha mantenido por encima de las demás categorías; sin embargo, se ha observado que al finalizar el periodo 2018 ha sido superada levemente por la categoría normal y deficiente.

Con respecto a la categoría Deficiente, se observa que ha tenido variaciones que han llegado por debajo de 10000 y por encima de los 70 000. Así mismo, la categoría normal ha mostrado menos variaciones y una tendencia creciente, la cual, ha superado a la categoría Deficiente en enero del periodo 2018; la categoría normal inició en enero del periodo 2017 con un monto de 29 070,24 y culminó el periodo 2018 con un monto de 62 731,68.

Por otra parte, las provisiones en la categoría de CPP y Pérdida han variado levemente y se han mantenido por debajo 10000 soles, a excepción de la categoría de CPP en octubre del periodo 2018.

\section{ANÁLISIS DE LA CARTERA DE RIESGO POR TIPO DE CRÉDITO}

\section{Cartera de Riesgo de los Créditos a grandes empresas}

Los resultados de la figura 8, muestran que los Créditos a grandes empresas; la categoría CPP se elevó en marzo y bajó completamente en julio del periodo 2018; la cartera Dudoso subió en junio y bajó completamente en septiembre del periodo 2018; la cartera Deficiente subió en julio y bajó completamente en noviembre del periodo 2018; la cartera Normal subió a 30,7\% en marzo del periodo 2018, pero en diciembre del mismo periodo bajó al $15,7 \%$. La cartera de Pérdida, no se elevó durante los periodos.

\section{Cartera de Riesgo de los Créditos a medianas empresas}

Los resultados de la figura 9, muestran que los Créditos a medianas empresas; donde, la cartera Normal se mantuvo entre el $70 \%$ y $80 \%$, pero en septiembre del periodo 2018 se redujo la cartera y se mantuvo entre el $60 \%$ y $70 \%$. La cartera Pérdida está entre el $10 \%$ y el 20\%. Así mismo, cuando la cartera Normal bajó la cartera CPP se elevó, luego se elevó la cartera Deficiente y luego la cartera Dudoso mostrando una reducción en la calidad de cartera. Cabe resaltar que la mayoría de las carteras no mostraron cambios importantes.

\section{Cartera de Riesgo de los Créditos a pequeñas empresas}

Los resultados de la figura 10, muestran que los Créditos a pequeñas empresas; donde, la cartera Normal se mantuvo entre el $80 \%$ y $90 \%$. El resto de carteras se mantuvo por debajo del 10\%; lo cual es muestra de una buena gestión. 


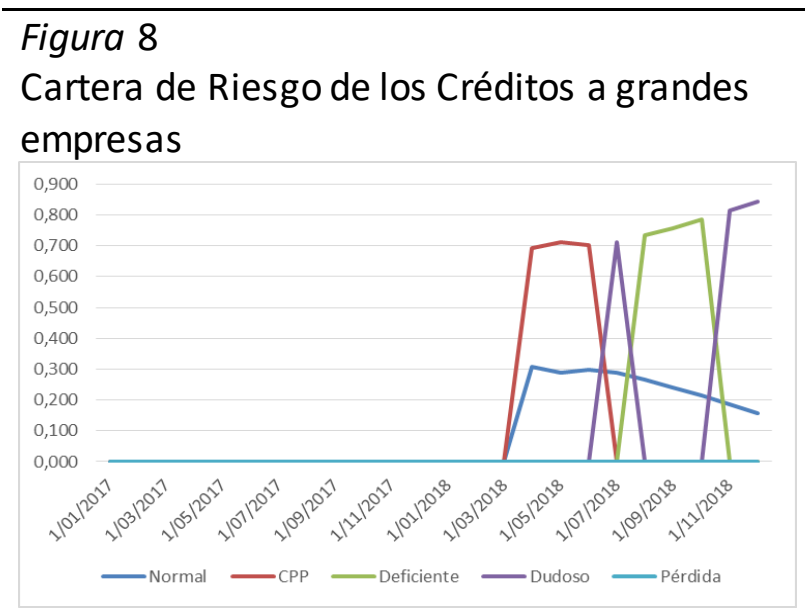

Figura 2

Cartera de Riesgo de los Créditos a pequeñas empresas

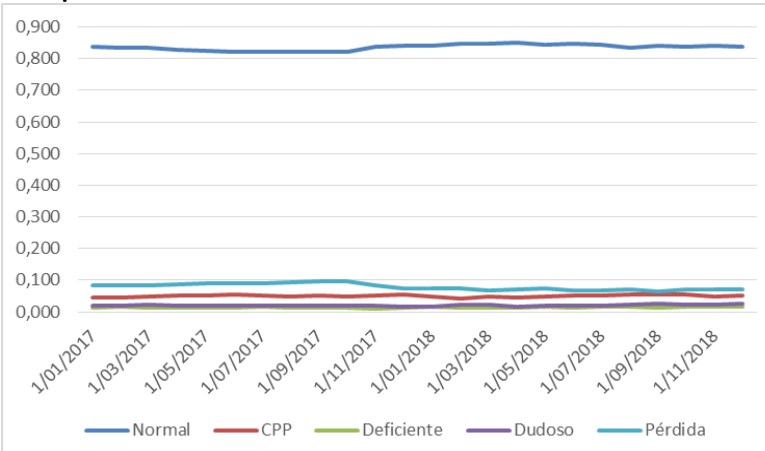

Figura 3

Cartera de Riesgo de los Créditos de consumo

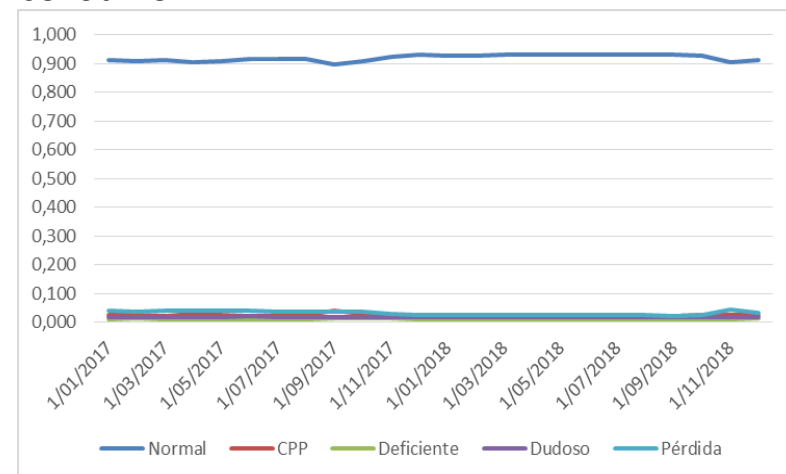

Figura 1

Cartera de Riesgo de los Créditos a medianas empresas

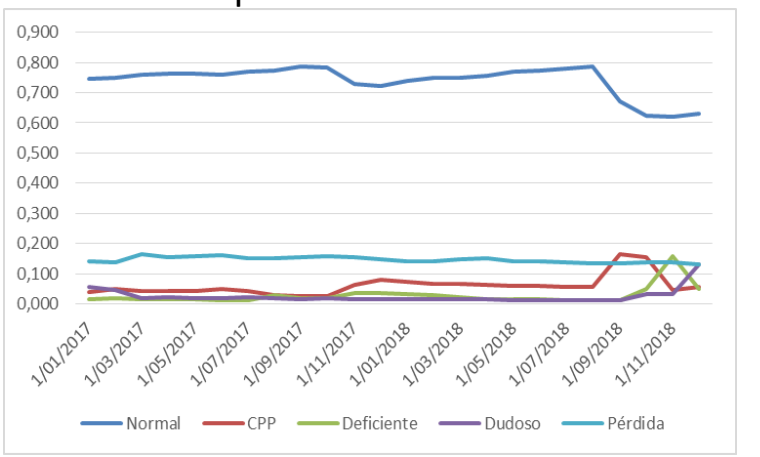

\section{Figura 11}

Cartera de Riesgo de los Créditos a microempresas

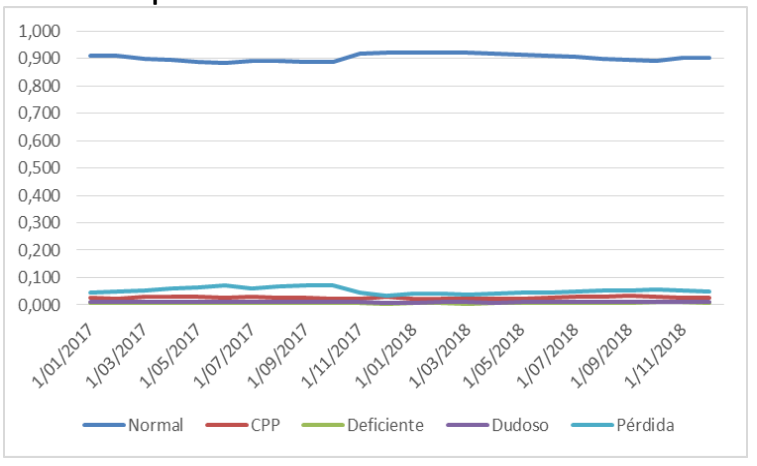

Figura 4

Cartera de Riesgo de los Créditos hipotecarios para vivienda

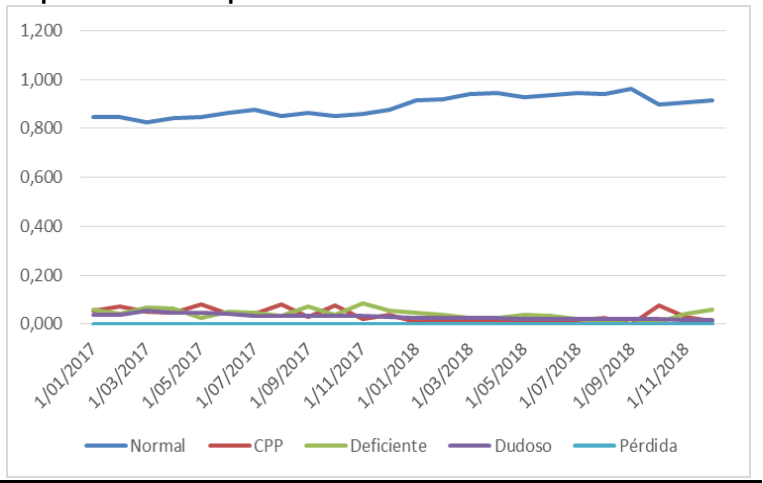

\section{Cartera de Riesgo de los Créditos a microempresas}

Los resultados de la figura 11, muestran que los Créditos a microempresas; donde, la cartera Normal se mantuvo en el $90 \%$ con leves variaciones. El resto de carteras se mantuvo por debajo del 10\%; lo cual es muestra de una buena gestión, similar a los resultados de los Créditos a pequeñas empresas. 


\section{Cartera de Riesgo de los Créditos de consumo}

Los resultados de la figura 12, muestran que los Créditos de consumo; donde, la cartera Normal se mantuvo levemente por encima del $90 \%$, con leves variaciones. El resto de carteras se mantuvo por debajo del $10 \%$; lo cual es muestra de una buena gestión, similar a los resultados de los Créditos a pequeñas empresas y micro empresas.

\section{Cartera de Riesgo de los Créditos hipotecarios para vivienda}

Los resultados de la figura 13, muestran que los Créditos hipotecarios para vivienda; donde, la cartera Normal se mantuvo entre el $80 \%$ y $96 \%$, con leves variaciones. El resto de carteras se mantuvo por debajo del 10\%; lo cual es muestra de una buena gestión, similara los resultados de los Créditos a pequeñas empresas, micro empresas y de consumo.

\section{ANÁLISIS DE LAS COLOCACIONES POR TIPO DE CRÉDITO}

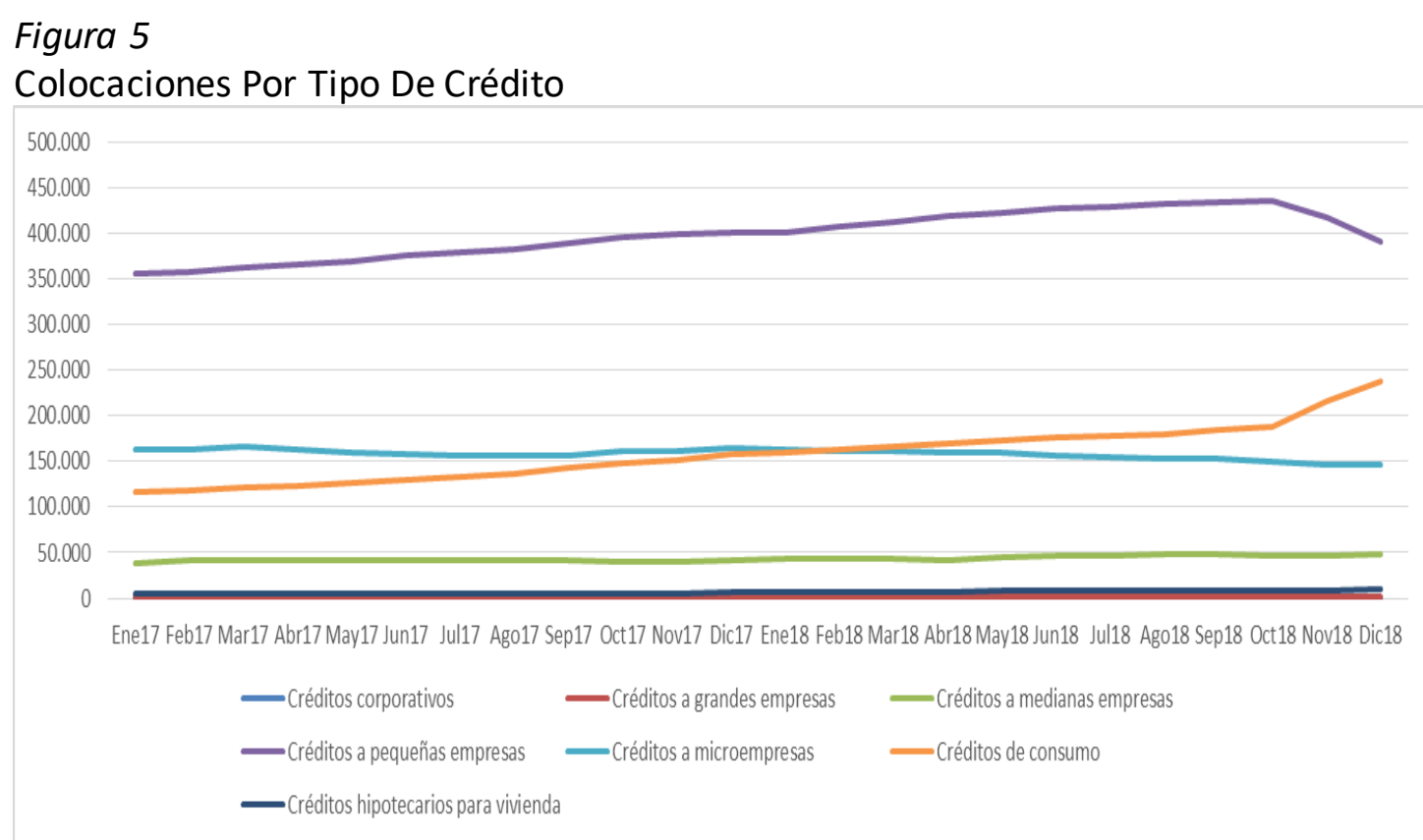

Los resultados de la figura 14, muestra que el mayor número de colocaciones se encuentra en los Créditos a pequeñas empresas, la cual inició por encima de 350 millones, superó los 400 millones; sin embargo, en octubre del 2018, mostró una tendencia descendente que se mostró por debajo de los 350 millones al culminar el periodo 2018. Los Créditos a microempresas iniciaron el periodo 2017 por encima de los 150 millones, solo mostró una tendencia a la baja al final del periodo 2018 al mostrarse por debajo de los 150 millones. Los créditos de consumo dieron un gran salto, porque al iniciar el periodo 2017 se ubicaba por encima de los 100 millones; en octubre del periodo 2018 los Créditos de consumo está cercano a los 150 millones, luego al finalizar el periodo 2018 los Créditos de consumo se elevaron muy cerca a los 250 millones. 
Por otra parte, los Créditos a grandes empresas y los Créditos hipotecarios para viviendas, se mantuvieron por debajo de los 50 millones y no mostraron cambios significativos.

\section{ANÁLISIS DE LA VARIACIÓN DEL PBI INFLACIÓN Y EMPLEO}

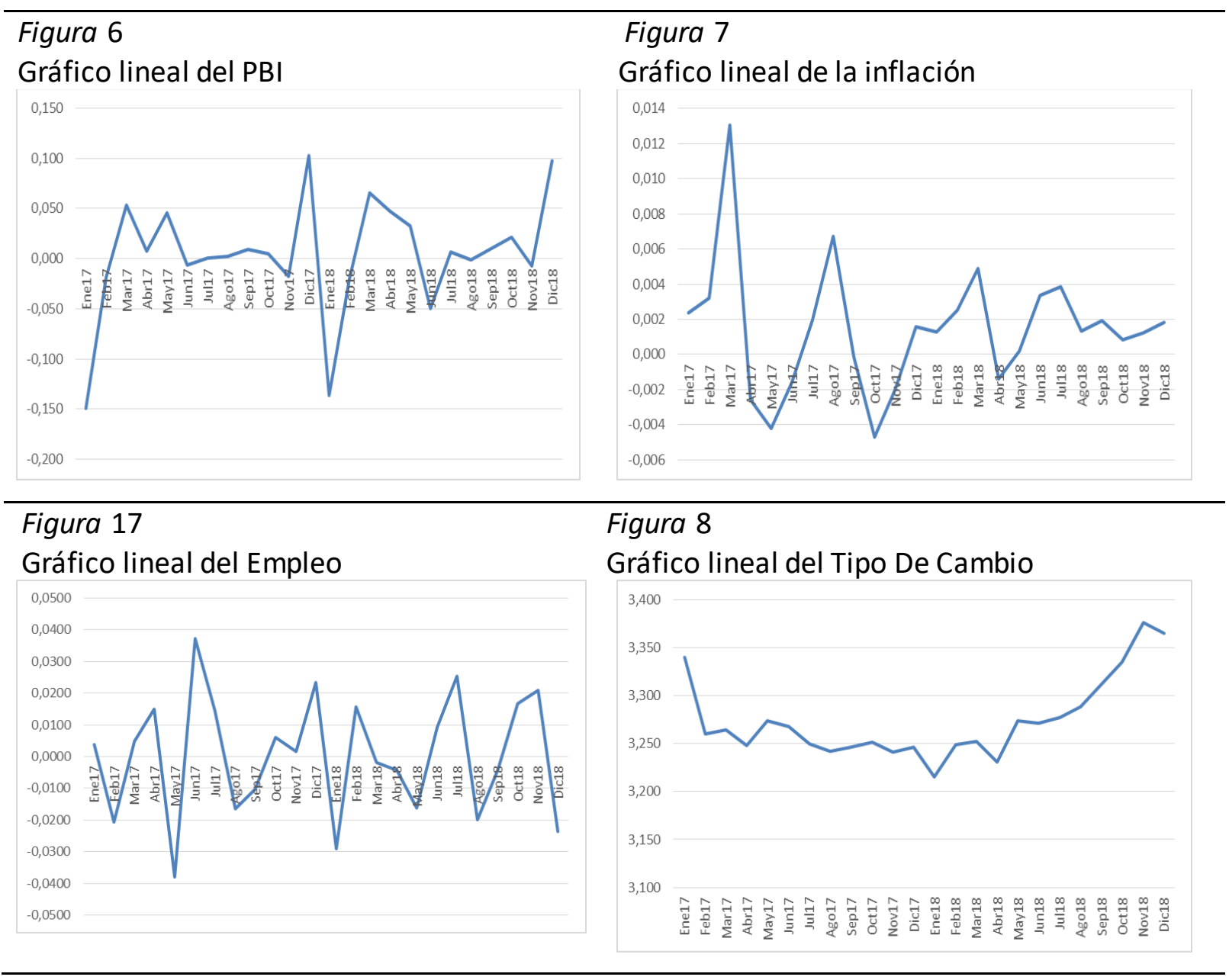

Los resultados de la figura 15, muestran las variaciones del PBI, durante los periodos evaluados; se puede observar que no hay una tendencia clara, se podría afirmar que inició el periodo 2017 con una variación negativa y que al finalizar el periodo 2018 mostró una variación positiva. Mostrando el pico más alto en diciembre del periodo 2017 y el valle más bajo en enero del periodo 2017. Es preciso destacar que luego del pico en diciembre del 2017 se mostró un valle en enero del periodo 2018, luego, durante febrero y marzo se dio una recuperación en cuanto la variación del PBI.

Los resultados de la figura 16, muestran las variaciones de la Inflación, durante los periodos evaluados; se puede observar que las principales variaciones se dieron al iniciar el periodo 2017, sin embargo, dichas variaciones se redujeron durante el periodo 2018.

El pico más alto se dio en marzo y el valle más bajo se dio en octubre del periodo 2017. Cabe resaltar, que una menor variación podría serfruto de una buena gestión por parte de los organismos del Estado Peruano. 


\section{ANÁLISIS DE LA VARIACIÓN DEL EMPLEO}

Los resultados de la figura 17 muestran las variaciones del empleo; no es posible indicar que existe una tendencia clara. Se observa que al iniciar el periodo 2017, la variación ascendía a 0,0038; sin embargo, dichas variaciones se redujeron al culminar el periodo 2018 , con una variación que ascendió a -0,0236. El valle más bajo se dio en mayo y el pico más alto se dio en junio del periodo 2017. Es posible afirmar que la mayoría de variaciones fueron positivas.

\section{ANÁLISIS DEL TIPO DE CAMBIO}

Los resultados de la figura 18, muestran el Tipo De Cambio durante los periodos 2017 y 2018. Se observa que la variable muestra una tendencia similar a una curva parabólica convexa; es decir, al iniciar el periodo 2017, el Tipo De Cambio ascendía a 3,340; sin embargo, se redujo a través del tiempo a 3,215; y finalizó el periodo 2018 con un valor de 3,364. El valle más bajo se dio en enero y el pico más alto se dio en noviembre del periodo 2018. Las variaciones encontradas fueron pequeñas.

\section{Rentabilidad}

\section{ANÁLISIS DEL ROA - ROTE - ROE}

Figura 18

Gráfico lineal del ROA - ROTE - ROE

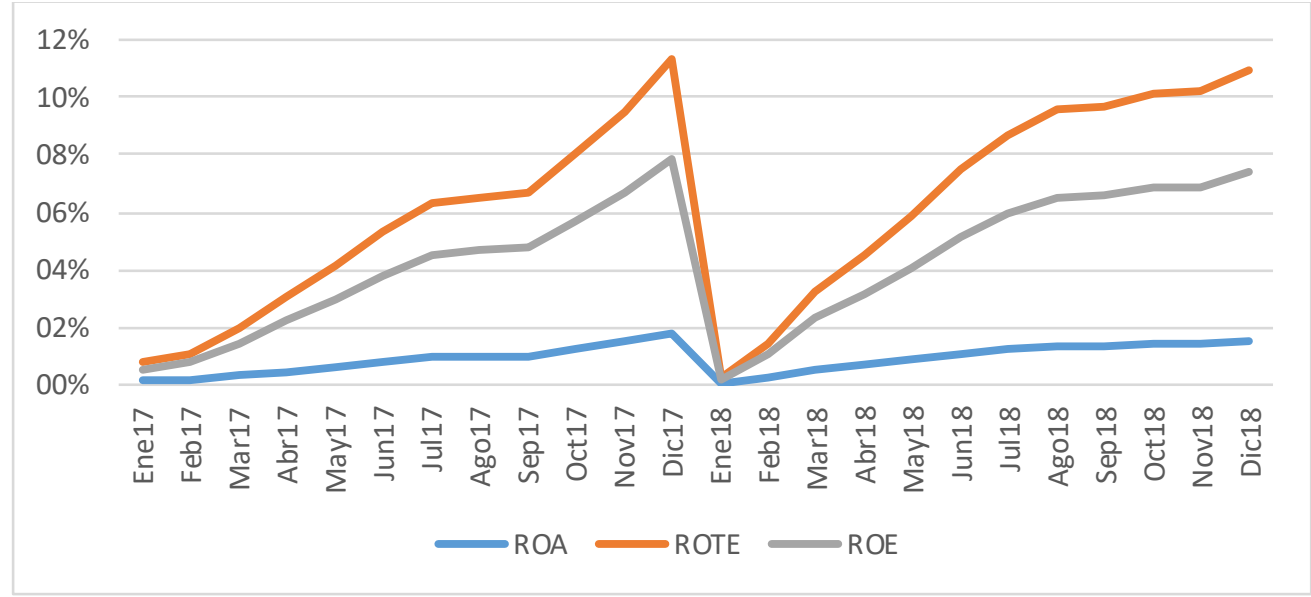

Los resultados de la figura 18, muestran el ROA - ROTE - ROE en el periodo estudiado. Se observa que el movimiento de las curvas son similares; sin embargo, debido a los componentes de los ratios, la ROTE está por encima del ROE, a la vez, el ROE está por encima del ROA; esto significa que la empresa está financiando sus activos con una deuda que supera la rentabilidad económica.

Es posible observar que la rentabilidad inició muy cerca de $0 \%$ al iniciar el periodo 2017 , elevándose hasta diciembre del periodo 2017 y cayendo estrepitosamente; dicha tendencia fue similar al iniciar y finalizar el periodo 2018. 


\section{DISCUSIÓN}

Con respecto al recojo de los datos, se consideró pertinente trabajar con los periodos 2017 y 2018, porque año tras año los escenarios en el sector económico - financiero son muy diferenciados; además, con 24 datos es posible realizar correlaciones aceptables. Entre las principales limitaciones, resalta la normalidad de las variables, especialmente entre los factores relacionados a la calidad de cartera de créditos; porque debido a ello, variaron las pruebas estadísticas. Por otra parte, algunas de las variables en estudio no contaban con una correlación y por ello, no fue posible realizar una prueba para hallar la influencia de una variable en otra.

No existe un alto grado de influencia de la Morosidad por tipo de crédito en la rentabilidad de la Caja Municipal de Ahorro y Crédito De Tacna S.A., periodo 2017 - 2018. Dicho resultado, puede relacionarse con el cambio brusco de la Morosidad de los Créditos a grandes empresas durante algunos meses. Los resultados de la variable Morosidad, son diferentes a los obtenidos por Acosta \& Sánchez (2016); quien resalta en su investigación que, entre los factores determinantes de la rentabilidad, se encuentran: la cartera de riesgo, gestión de créditos, índices de morosidad, gastos en provisiones.

A pesar que, no se obtuvo una correlación de todos los componentes, se halló que existe una correlación significativa entre la Morosidad de Créditos a Grandes Empresas y la rentabilidad, dicha correlación fue positiva y moderada. También, se halló que existe una correlación significativa entre la Morosidad de Créditos a Medianas Empresas y la rentabilidad, dicha correlación fue positiva y moderada. A la vez, estos resultados son contrastados con Calloapaza (2017), quien asegura que, la morosidad es el principal factor de la calidad de cartera de créditos; situación descrita por Martínez (2006), quien indicó que los índices elevados de morosidad afectan directamente la rentabilidad y utilidades de la organización. Murrugara \& Ebentreich (2009) por su parte, dio a conocer que el factor interno vinculado a la calidad de cartera de créditos es la morosidad, además es el indicador más usado en este análisis; también descrito por la investigación de (Aguilar \& Camargo, 2002).

No existe un alto grado de influencia de las Provisiones por tipo de crédito en la rentabilidad de la Caja Municipal de Ahorro y Crédito De Tacna S.A. Es preciso mencionar que las provisiones para los créditos diferentes a los de la categoría normal, varían de mes a mes porque los clientes son categorizados de acuerdo a los días de morosidad, algunos vuelven a la categoría de normal y pagan sus deudas, en otros casos se aplica la cobranza coactiva para la recuperación del crédito. Los resultados obtenidos en esta variable, de alguna forma contrastan con los resultados obtenidos por Cornejo (2017), quien halló una relación inversa entre las provisiones y la rentabilidad de las Cajas Municipales de Ahorro y Crédito en el Perú; cabe señalar que, al tomar un mayor conjunto de datos, tanto de periodos como de entidades, es posible reducir las desviaciones y normalizar los datos.

No existe un alto grado de influencia de la Cartera de Riesgo en la Rentabilidad de la Caja Municipal de Ahorro y Crédito De Tacna S.A., periodo 2017 - 2018. Este resultado, se vincula con la gran variabilidad mostrada por la Cartera de Riesgo de los Créditos a grandes empresas, mostrada a partir de marzo del periodo 2018; en las demás carteras se observó el predominio de la cartera Normal.

Existe un alto grado de influencia de las Colocaciones por tipo de crédito en la rentabilidad de la Caja Municipal de Ahorro y Crédito De Tacna S.A. Así mismo, el mayor número de colocaciones se encuentra en los Créditos a pequeñas empresas. Estos resultados 
concuerdan con los resultados por Gomez (2011), quien señala que la cartera de créditos crece con el número de colocaciones y por lo tanto, con la rentabilidad. Aparicio \& Moreno (2011), dio a conocer a través de su investigación que la cartera de consumo es la más sensible a los efectos de las variables externas como las macroeconómicas, en ese sentido, se halló que existe una correlación del $29,29 \%$ entre el PBI y los créditos de consumo; tal como lo mencionaron los autores.

Las variaciones del PBI, fueron negativas y positivas durante el periodo evaluado; cabe resaltar que las variaciones fueron mayores al iniciar y culminar los periodos. Con estos resultados, se podría afirmar que no se ha obtenido suficiente información para corroborar lo planteado por Aparicio \& Moreno (2011), quienes vinculan la calidad de cartera con el PBI, esta calidad, relacionada directamente con la rentabilidad de la entidad.

No existe un alto grado de influencia de la Inflación en la rentabilidad de la Caja Municipal de Ahorro y Crédito De Tacna S.A. Cabe resaltar que, la inflación fue mayor al inicio de los periodos y más adelante se redujo de forma paulatina; sin embargo estas variaciones no coincidieron con la rentabilidad de la entidad. Cabe resaltar que Muñoz (1999) afirma que la presencia de una elevada inflación incrementaría notoriamente la probabilidad de problemas sistémicos, vinculados a la calidad de cartera y por lo tanto, en la rentabilidad.

La variable Empleo, no mostró tendencia alguna y no coincidió con las variaciones de la rentabilidad. Así mismo, no se halló suficiente información para afirmar que el empleo influye principalmente en la capacidad de pago de los prestatarios y por lo tanto en el pago de sus deudas, para la rentabilidad de la sostenibilidad de la entidad; como afirma De Gregorio (2012).

No existe un alto grado de influencia del Tipo de cambio en la rentabilidad de la Caja Municipal de Ahorro y Crédito De Tacna S.A., periodo 2017 - 2018. La nula relación entre las variables, tiene que ver con la dación de créditos, la cual en su mayoría se brinda es soles, no afectando la rentabilidad de la entidad. Blanchard \& Enrri (2000) explica como el tipo de cambio afecta el sistema financiero de todo el país; sin embargo, esto no se refleja en los resultados obtenidos dentro de la investigación, así mismo, gran parte de los créditos brindados por la entidad, se dan en soles y no en dólares.

Así mismo, los resultados de las pruebas de hipótesis con las variables macroeconómicas, muestran que dichas estas variables no se relaciona con los resultados individuales de la entidad; sin embargo, se cree que sí se hallaría algún tipo de relación si se ampliara el estudio, a todo el sistema financiero del Perú.

Con respecto a la hipótes is general, debido al comportamiento de los datos no se pudo realizar una regresión lineal, pero queda la posibilidad de aplicar una regresión multivariada que permita agrupar las variables dentro de un modelo. Si se desarrolla un modelo quitando algunas variables, el aporte del modelo no sería sustancial. Por otra parte, los factores que sí tuvieron influencia significativa en la rentabilidad fueron: provisiones y colocaciones.

De acuerdo a los resultados obtenidos, se recomienda a la entidad, implementar más cargos de control del riesgo de crédito que permita un mejor monitoreo y manejo de la morosidad. Evitar el sobre endeudamiento. Se debe evitar en lo posible, el deterioro de las carteras, especialmente en los Créditos a medianas empresas, pequeñas empresas, micro empresas, y de consumo. Constituir provisiones voluntarias que permitan cubrir la cartera problema por lo menos al $100 \%$, con el consecuente impacto favorable en la exposición 
patrimonial. Monitoreo constante para evitar el desplazamiento de la cartera mediante el sistema de cobranzas. Implementar alertas en base a las cuentas más representativas de riesgo. Propiciar el lanzamiento de campañas y/o productos crediticios de acuerdo a la realidad de cada zona. Se recomienda a la entidad, realizar proyecciones para pronosticar algún tipo de inflación futura en los diferentes sectores productivos; no solo porque a los clientes se les haría más difícil pagar un crédito, la entidad debe manejar diversos escenarios y debe contar con una persona encargada de darle seguimiento. El empleo, es una variable que sí afectaría el pago de los créditos brindados; en ese sentido, la ciudad de Tacna, a diferencia de gran parte de los departamentos del Perú, cuenta con una tasa de crecimiento promedio a nivel nacional; sea en el sector público y privado; así mismo, al ser una zona fronteriza goza de beneficios económicos, como el comercio; este último aporte en gran medida a muchas familias. Se recomienda a la entidad evaluar la posibilidad de internacionalizar sus servicios (considerando la ubicación fronteriza) de esta manera, atenuar la exposición al tipo de cambio y diversificar dicho riesgo.

\section{CONCLUSIONES}

No existe un alto grado de influencia del Tipo de cambio en la rentabilidad de la Caja Municipal de Ahorro y Crédito De Tacna S.A., periodo 2017 - 2018. Los activos en moneda extranjera están representados en mayor parte después del disponible, por la cartera de créditos \$US 2,9 millones, por esta razón la CMAC Tacna, con la finalidad de mitigar la exposición en moneda extranjera, decidió reducir las colocaciones en moneda extranjera desde el primer semestre del 2017, logrando mantener en niveles adecuados la fluctuación por tipo de cambio en la cartera crediticia.

\section{REFERENCIAS BIBLIOGRÁFICAS}

Acosta, S. E., \& Sánchez, B. C. (2016). Calidad de cartera de clientes para mejorar la rentabilidad de la Caja Municipal de Ahorro y Crédito Trujillo S.A. sucursal Lambayeque -2015. Recuperado de http://repositorio.uss.edu.pe/handle/uss/3113

Aguilar, G., \& Camargo, G. (2002). Análisis de la morosidad en las instituciones microfinancieras (IMF) en el Perú. Economía, 25(50), 65-121.

Aparicio, C., \& Moreno, H. (2011). Calidad de la cartera crediticia bancaria y el ciclo económico: Una mirada al gasto en provisiones bancarias en el Perú (2001-2011). Recuperado de http://www.sbs.gob.pe/Portals/0/jer/ddt_ano2011/3_Aparicio_y_Moreno_2011.pdf

Arriaza, M. (2006). Guía práctica de análisis de datos. Instituto Andaluz de Investigacion y Formacion Agraria, Pesquera, Alimentaria y de la Producción.

http://www.bancaynegocios.com/el-roa-y-el-roe-su-significado-y-como-calcularlo/

BCRP. (2011). Glosario de Términos Económicos. Recuperado de http://www.bcrp.gob.pe/docs/Publicaciones/Glosario/Glosario-BCRP.pdf

BCRP, B. C. de R. del P. (2018). Reporte de Estabilidad Financiera. Recuperado de http://www.bcrp.gob.pe/docs/Publicaciones/Reporte-Estabilidad-Financiera/refmayo-2018.pdf

BCRP, B. C. de R. del P. (2019). Reporte de Estabilidad Financiera. Recuperado de http://www.bcrp.gob.pe/docs/Publicaciones/Reporte-Estabilidad-

Financiera/2019/mayo/ref-mayo-2019.pdf 
Blanchard, O., \& Enrri, D. P. (2000). Macroeconomía: Teoría y política económica con aplicaciones a América Latina. Pearson Educación.

Calloapaza, C. A. (2017). El Comportamiento de la Morosidad de la CMAC Tacna S.A. y su Incidencia en los Resultados de la Gestión Crediticia, en las Agencias de Tacna Periodo 2010-2014. Recuperado de http://localhost:8080/xmlui/handle/UPT/486

Cornejo, C. G. (2017). Influencia de las Provisiones en la Rentabilidad de las Cajas Municipales de Ahorro y Crédito en el Perú, Periodo 2011-2016. Recuperado de http://localhost:8080/xmlui/handle/UPT/290

De Gregorio, J. E. (2012). Macroeconomia Intermedia. Pearson Educación.

Equilibrium, E. C. de R. S. A. (2018). Caja Municipal de Ahorro y Crédito de Tacna (CMAC Tacna). Recuperado de http://www.equilibrium.com.pe/CmacTacnadic17.pdf

Equilibrium, E. C. de R. S. A. (2019). Caja Municipal de Ahorro y Crédito de Tacna (CMAC Tacna). Recuperado de http://www.equilibrium.com.pe/CmacTacna.pdf

Giraldo, W. (2010). Determinantes de la morosidad de la cartera en el sistema financiero Colombiano.

Recuperado

de http://repository.icesi.edu.co/biblioteca_digital/handle/10906/5394

Gomez, I. (2011). Analisis del indice de morosidad de la cartera de clientes y su incidencia en la rentabilidad de la caja Piura-Agencia Huamachuco años 2006-2010. Recuperado de http://dspace.unitru.edu.pe/handle/UNITRU/3763

Hernández, R., \& Mendoza, C. P. (2018). Metodología de la investigación: Las rutas cuantitativa, cualitativa y mixta. McGraw-Hill Education.

INEI, I. N. de E. e I. (2014, mayo 13). Glosario de Términos. Recuperado de https://www.inei.gob.pe/media/MenuRecursivo/publicaciones_digitales/Est/Lib1154 /glosario.pdf

Instituto Nacional de Estadística e Informática. (2018). Perú: Indicadores de Empleo e Ingres o por departamento. Recuperado de https://www.inei.gob.pe/media/MenuRecursivo/publicaciones_digitales/Est/Lib1537 /libro.pdf

Lozano, M. M. (2007). ¿La eficiencia del personal en las microfinancieras influye en la cartera vencida? Análisis Económico, XXII(50), 173-184.

Merchán, L. A. (2016). ¿Afecta la distancia de residencia a los centros urbanos la calidad en la cartera de créditos? Caso aplicado a una entidad financiera de Colombia. Recuperado de http://repository.javeriana.edu.co/handle/10554/20410

Morales, A., Morales, J. A., \& Alcocer, F. R. (2014). Administración Financiera. Grupo Editorial Patria.

Morales, J., \& Tuesta, P. (1999). Calificaciones de crédito y riesgo país. Banco Central de Reserva del Perú.

Muñoz, J. (1999). Calidad de cartera del sistema bancario y el ciclo económico: Una aproximación econométrica para el caso peruano. BCRP Estudios Económicos. Recuperado de https://core.ac.uk/download/pdf/7115856.pdf

Murrugara, E., \& Ebentreich, A. (2009). Determinantes de morosidad en entidades de microfinanzas: evidencia de las EDPYMES. Recuperado de http://www.sbs.gob.pe/Portals/0/jer/EDIPUB_VOLUMEN1/Murrugarra.pdf

RENACYT. Reglamento de calificación, clasificación y registro de los investigadores del sistema nacional de ciencia, tecnología e innovación tecnológica. , Ley № $28613 \S$ (2005).

SBS. Resolución SBS N¹1356-2008. , (2010). 
SBS. (2015a). Glosario de Términos e Indicadores Financieros. Recuperado de https://intranet2.sbs.gob.pe/estadistica/financiera/2015/Setiembre/SF-0002se2015.PDF

SBS. (2015b). Glosario De Términos E Indicadores Financieros (p. 14). Recuperado de https://intranet2.sbs.gob.pe/estadistica/financiera/2015/Setiembre/SF-0002se2015.PDF

SBS. (2019a). Información Estadística de Cajas Municipales. Recuperado 5 de agosto de 2019, de Superintendencia de Banca, Seguros y AFP website: http://www.sbs.gob.pe/app/stats_net/stats/EstadisticaBoletinEstadistico.aspx?p=3\#

SBS. (2019b). Sistema Financiero. Recuperado 2 de febrero de 2019, de http://www.sbs.gob.pe/estadisticas/sistema-financiero

Supo, P. F. (2015). Factores que determinan la calidad de la cartera crediticia de la caja rural de Ahorro y Crédito los Andes - Agencia Ayaviri en el Período 2011 - 2014. Recuperado de http://repositorio.unap.edu.pe/handle/UNAP/2515

Vara, A. (2010). 7 pasos para una tesis exitosa (Segunda). Lima: Universidad San Martín de Porres.F 